\title{
Multi-spectral and Topographic Fusion for Automated Road Extraction
}

https://doi.org/10.1515/geo-2018-0036

Received September 5, 2017; accepted June 13, 2018

\begin{abstract}
Road geometry is pertinent information in various GIS studies. Reliable and updated road information thus calls for conventional on-site survey being replaced by more accurate and efficient remote sensing technology. Generally, this approach involves image enhancement and extraction of relevant features, such as elongate gradient and intersecting corners. Thus far, its implication is often impeded by wrongly extraction of other urban peripherals with similar pixel characteristics. This paper therefore proposes the fusion of THEOS satellite image and topographic derivatives, obtained from underlying Digital Surface Models (DSM). Multi-spectral indices in thematic layers and surface properties of designated roads were both fed into state-of-the-art machine learning algorithms. The results were later fused, taken into account consistently leveled road surface. The proposed technique was thus able to eliminate irrelevant urban structures such as buildings and other constructions, otherwise left by conventional index based extraction. The numerical assessment indicates recall of $84.64 \%$, precision of $97.40 \%$ and overall accuracy of $97.78 \%$, with 0.89 Kappa statistics. Visual inspection reported herewith also confirms consistency with ground truth reference.
\end{abstract}

Keywords: road extraction; spectral indices; data fusion; DSM; THEOS

\section{Introduction}

Computerized Remote Sensing (RS) technology has presently been adopted in various fields, ranging from geographical environment, hydrology to ecology and other administrative tasks, tackling both urban and rural issues. These include but not limited to transportation and

Supattra Puttinaovarat: Ph.D., Prince of Songkla University, Surat Thani Campus, Thailand

*Corresponding Author: Paramate Horkaew: Suranaree University of Technology Muang, Thailand, E-mail: phorkaew@sut.ac.th navigation, logistic distribution route scheduling, urban planning and growth analysis, public hazard mitigation, and natural resource management, etc. Existing studies generally approached these issues by first extracting objects of interest from remotely sensed maps [1-3], with respect to underlying land covers and/ or other man-made structures (e.g., buildings, constructions and roads, etc.) [4], and then analyzing their shape characteristics. Among many other structures, road is considered one of the most crucial requisites for subsequent geographical analyses, since it serves as a connective passage of concerning elements. Reliable and most updated details on roads network and their geometrical properties are important to ensure informed planning. A government takes into account road information, for instances, in allocating resources, devising appropriate urban planning, and preparing contingency evacuation routes, etc. [5, 6]. The enterprise sectors also rely on road network for designing their economically efficient logistic strategy [7]. In addition to these institutional interests, individuals may well benefit from these information for navigating them to unfamiliar locations via most economic or fastest routes [8]. Accordingly, without the most recent and reliable road data, these provisions cannot be met so effectively and as such traditional on-site human survey, despite its accuracy, has no longer been an efficient means of serving such information.

Satellite imaging has increasingly become much accepted alternative. Extracting roads or classifying their pixels from satellite images has advantages of being apparently accurate and most up to date. In addition, the modality is more cost effective, compared to conventional expedition, in terms of both human resource and expenses. Applying computerized techniques for analyzing these images could potentially lessen requirement for interpretation by an expert. According to recent literature, there were five imaging modalities employed in this scenario, i.e., satellite, radar, aerial, Lidar and digital terrain images. Nonetheless, superior extractions obtained from high resolution satellite images, e.g., WorldView, QuickBird, SPOT, IKONOS and THEOS, etc., [9-11] have made them prevailing. Computerized road extraction methods can be categorized into two groups, that are fully- and 
semi-automatic, depending on whether or not a user is required to provide initialization within the area [4]. Typical techniques used to differentiate a road from surrounding pixels included mean shift, level set, Support Vector Machine (SVM) or shape features, etc. Concentrating on planar mapping, they share similar drawbacks, which are 1) operator having to adjust kernel sizes due to roads with varying width and 2) inability to subtract some non-roads due to ambiguous pixel hypotheses (e.g., local intensity and front/ boundary curvature, etc.). With the recent advances in sensors, there had been studies adopting multispectral indices in road extraction. They are NDBI [12], NDVI [13-18], SAVI [18-20] and NDWI [15, 21]. Theses indices are expressed as arithmetic ratio of multi-wavelength reflectance coefficients. Since these values are material specific, their statistics could be employed to highlight materials of interest. It remains yet challenging to meticulously set specific thresholds that are most suitable for versatile acquiring conditions and geographical areas. Consequently, some other urban structures are inevitably misinterpreted and classified as roads. There have been several attempts made to devise new road discriminating indices from WorldView-2 satellite data [22, 23]. The results reported therein similarly showed some false positives (buildings) as well as false negatives (incomplete roads).

THEOS satellite imaging has been another modality of interests in recent surveys. Pan-Sharpen image was employed in road extraction by using, for example, morphology and Fuzzy C-means for pre-processing and automated classification, respectively [24]. This study exhibited 70\% extraction accuracy, with error being false positive due to urban objects. To date, multi-spectral THEOS and other high resolution satellite images were yet unable to effectively differentiate road traces from other constructions, especially by means of reflectance indices, thanks to their limited spectral resolution $[25,26]$. To resolve this issue, while maintaining its relatively rich spatial details, this study proposes fusing multi-spectral THEOS with Digital Surface Model (DSM) data. With this approach, spectral indices will be integrated with local topographic derivatives, into vector attributes. Both supervised and un-supervised machine learning (ML) and fusion based on these vectors were then employed in order to accurately discriminate roads from other build-ups. The remaining of this paper is organized as follow: Section 2 reviews the related literature and materials in greater details, i.e., spectral indices, DSM, ML and data fusion. Sections 3 and 4 describes the proposed method and reports experimental results, both numerically and visually, with discussions. Finally, section 5 states the concluding remarks of this study, its merits and implication of the findings.

\section{Related Literature}

Data fusion has often been employed in remote sensing studies to verify and consolidate both acquired and processed information, normally from different imaging modalities. Fusion in post processing, for instances, was applied after land cover classification such as building [27], river [28] and road [16], etc. The two most prominent fusion methods are Majority Voting [29] and Dempster-Shafer [30]. The former was used to fused road extractions from LiDAR between using Minimum Distance (MD) and Maximum Likelihood (ML) [31], while the latter was used to fuse extracted roads from both LiDAR and aerial images [16]. Motivated by prior results, this study proposed data fusion between extracted roads based on THEOS spectral indices and those characterized by respective DSM derivatives. It will be later shown in our experiments that for this purpose, a more trivial and efficient Majority Voting sufficed.

Geographical fusion can operate on more than one hypotheses extracted from same or different modalities, which in our proposal are spectral indices and topographic data. Spectral indices have currently been adopted in various RS and GIS studies, e.g., vegetation analyses, drought and inundation management, irrigation planning, and urban and rural change detection, etc. [32, 33]. Particularly for road extraction, there have been a number of indices explored, such as NDBI [12], NDVI [13-18], SAVI [18-20] and NDWI [15, 21]. Most existing techniques defined road extraction problem as separating index pixels by some thresholds, which may be assigned empirically [14] or statistically (e.g., K-means or mean-shift, etc.) [19]. Since these methods were both observer and data dependent, and in addition, sensor calibration was performed on primary reflectance but not on algebraic indices, hence unique discrimination point hypothesis is not necessarily confirmed. Accordingly, there remained incomplete road segments or wrongly inclusion of irrelevant objects. To reduce ambiguity due to similar index ranges, some studies eliminated, for example, water surfaces by introducing a new index, derived from NIR2 band in a WorldView-2 image [22, 23]. Those works however remained unsuitable for urban area, where buildings and other constructions could be wrongly interpreted as roads. In addition, they relied primarily on NIR2 band, without which road specific indices could not be computed. It was evident from previous studies that, single index was not sufficient to differentiate roads from other objects.

Since reflective indices of the same structure may vary from one pixel to another, or those of different ones could 
be similar, an all-round optimal pixel-wise threshold may be futile. To elevate this problem, some studies thus incorporated shape scale and compactness and as such devised an object based classification (OBC). Based on this proposal, several modalities had so far been considered, with more promising results. OBC in SPOT5 and QuickBird yielded about $92-93 \%$ accuracy of road extraction, leaving a few buildings and other constructions [34, 35]. Similar approach was adopted in WorldView2 images, with $88 \%$ accuracy [36]. OBC was also combined with Fuzzy technique and applied to fused 3 satellite images, i.e., IKONOS 2, Quick bird, and WorldView2, however not so much as successfully, with only $72.36 \%$ accuracy [37]. SVM had similarly been considered [38], by using both IKONOS and QuickBird, but each in different study areas. In this work, $\mathrm{OBC}$ was found superior to pixel-wise classification. Some shape hypotheses had regardless to be imposed, resulting in large performance variability (as much as approximately 13\%) dependent on characteristics of study areas.

In addition to spectral indices derived from these satellite images, other investigations also considered DSM as an alternative or enhancement. DSM is a spatial model which expresses topographic elevation taken into account, for instances, canopy and building roofs. Since the measurements are obtained from an airborne sensor, the actual terrain heights are sometimes unavailable, especially in agricultural and urban areas. Nonetheless, this modality is most suitable for discriminating buildings and constructions [39, 40]. Some studies analyzed DSM and used it to determine flooded basin [41] or fused it with Landsat images to extract water bodies, passages and small branching [28]. Integrating DSM with aerial data was also proposed by Herumurti et al. in the recent road extraction studies, [42] and [43]. The basis of both works was the Radon transform, where the former and the latter adopted zero crossing strategy and morphology, respectively. The resultant accuracies of about $80 \%$ on average suggested that the Radon transform with the proposed preprocessing was superior to generic Hough transform and to relying on either DSM or aerial image alone. Taking data fusion approach, Rodriguez-Cuenca [16] purposed road extraction from an aerial image fused with LIDAR. Specifically, nDSM, derived from LIDAR by subtracting DTM from DSM, was used as a determinant. The rationale was that road absolute elevations varied from one route to another, it was hence not possible to specify a single value threshold to extract roads. Under normal circumstances, DSM height in open areas should be identical to that of DTM, while the numbers would differ if there exist, for instance, buildings, plants or roads, etc., on top of the terrain. This offset, namely nDSM, taken into account other heuristic at- tributes, could be used to identify land-cover types. Care should however be observed, since after subtraction, there may be parts of elevated ground terrain, confounded with the extracted roads. This averse effect was notable in their experiment. The extraction achieved in that study was $87.87 \%$ accurate, with a Kappa coefficient of 0.63. Based on similar DSM and nDSM data, SVM was employed as a supervised classifier in yet another independent study [44], where the extraction accuracy of $63.7 \%$ was reported.

In land cover extraction, it is evident that most of the previously mentioned studies relied their automation heavily on Machine Learning (ML) strategies. ML is a computerized method that has extensively been applied in remote sensing, especially in extracting and in classifying land-use and land-cover, such as determining water reservoir, construction and building, and road. ML in the literature can be categorized into supervised and unsupervised techniques. They were, for instances, SVM [45], ANN [33], KNN [46], K-mean [47] and Fuzzy C-means [48], etc. For road extraction, most works typically applied an ML classifier to pre-processed pixels. Wang et al. [49], for instances, applied K-mean to median filtered QuickBird images, while Maurya et al. [50] applied Fuzzy Cmeans to pan-sharpen THEOS images. Despite relatively high accuracy of $70-85 \%$, these works had suffered from over-segmentation of inadmissible structures, whose pixel intensities overlapped those of roads. Discrimination on hyper-planar curves such as SVM has thus been a preferred choice, according to recent surveys. Surface heights from DSM and nDSM, for example, were used as SVM input vectors [16]. Based on Gabor responses of HSV images, Matkan et al. [45] compared SVM and K-mean and founded that former was more accurate. Thus far, the extracted roads remained unstructured and fuzzy, and thus unsuitable for subsequent computerized analysis. To reduce false detections in SVM, another study [24] employed ad-hoc structural and morphological post-processing for non-road removals. Insights into these studies further reveal that SVM is suitable for multi-classes data with curvilinear discriminations. Its accuracy supersedes other supervised methods, even data distribution is non-Gaussian and especially when only a small training set is available. This study therefore chose the SVM in spectral indices based classification. As for the geometrical derivatives however, more efficient K-means was sufficient to separate road from nonroad objects, without requiring expert supervision and also with much less computational demand. 


\section{Proposed Scheme and Experiments}

The main objective of this study was to improve both accuracy and robustness of road identification by means of THEOS and DSM data fusion. Both supervised and unsupervised ML strategies were employed to overcome impeding factors reported in the related literature. More specifically, three spectral indices of road and non-road appearance were trained by using SVM under expert supervision, while their respective standard deviation of local surface were trained by unsupervised K-mean algorithm. The extracted road from both strategies were then fused by means of Majority Voting. Compared to classical pixel-wise processing, SVM was empirical and hence less prone to inter and intra-observer variabilities. The reason for this was that, unlike near field X-ray imaging for example, reflective indices were not calibrated per geographical location and atmospheric parameters. Determining an optimal threshold for roads had to rely on their visual cue, which in contrast to our case where they were implicitly learnt by SVM. Subsequently, corresponding a priori on local surface properties learnt by K-means reinforced the actual roads, while removing any sporadic extraction occurred across supporting vector boundaries, due to ambiguous distribution. The proposed scheme is summarized in Figure 1.

\subsection{Studied Area}

In this paper, the studied area was Muang district of Pathumthani province. It is located in the central region of Thailand. (Latitude $14^{\circ} 6^{\prime}$ North, Longitude $100^{\circ} 37^{\prime}$ East). The city is outgrowth of urban expansion from Bangkok metropolitan. Its notable characteristic is that its land uses constitute of extensive residential, commercial and industrial area developments, as well as rapidly growing logistics and transportation networks. These factors have contributed to the city being easily accessible, hence making it a prominent business center in the region. Accurately and reliably determining infrastructures linkage, especially its roads networks, was therefore of great interest, and as such was chosen for validating the proposed scheme.

\subsection{Data Preparation and Preprocessing}

Remote sensing data employed in this study were THEOS satellite images obtained from Geo-Informatics and Space

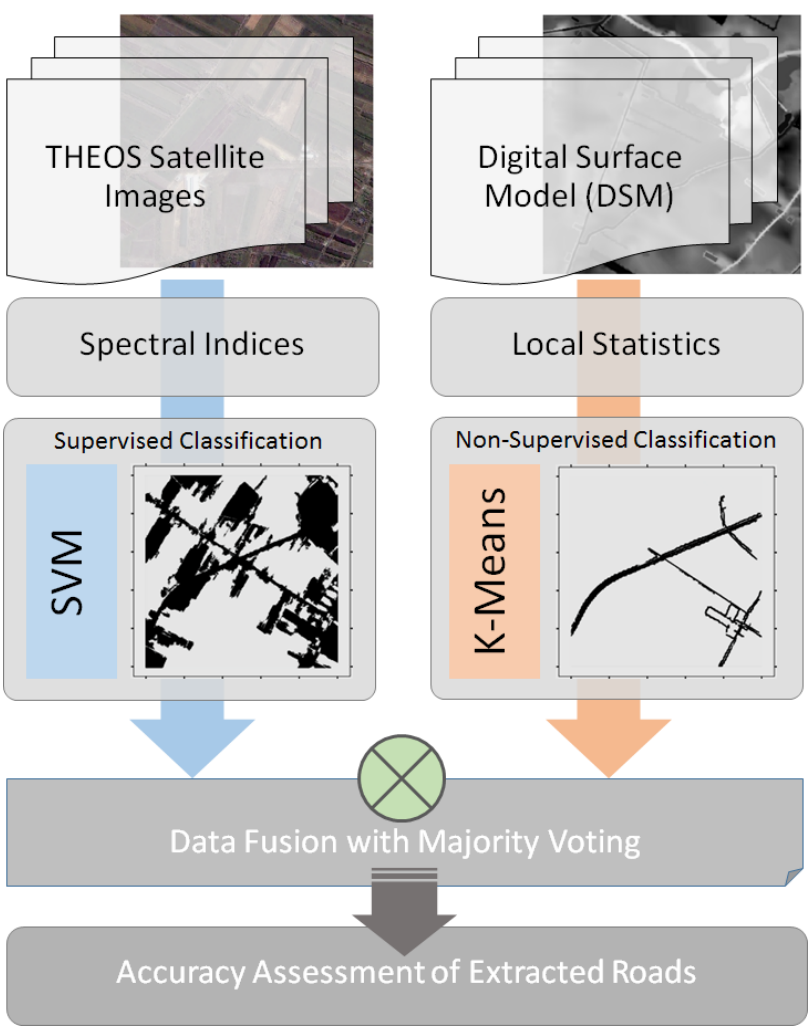

Figure 1: The flow diagram of the proposed scheme

Technology Development Agency (GISTDA), and Digital Surface Model (DSM) obtained from Land Development Department and Department of Public Works and Town \& Country Planning. Manually segmented roads ground truth for validation was also obtained from the latter institutes. Since multi-spectral THEOS images were recorded on $1^{\text {st }}$ February 2012 with a resolution of 2 meters, while that of DSM was only 5 meters, the DSM was first bi-linearly interpolated so that their spatial resolutions matched. THEOS acquisition details, including their spectral characteristics, were listed in Table 1. Figures 2 and 3 depict examples of THEOS and DSM data, respectively.
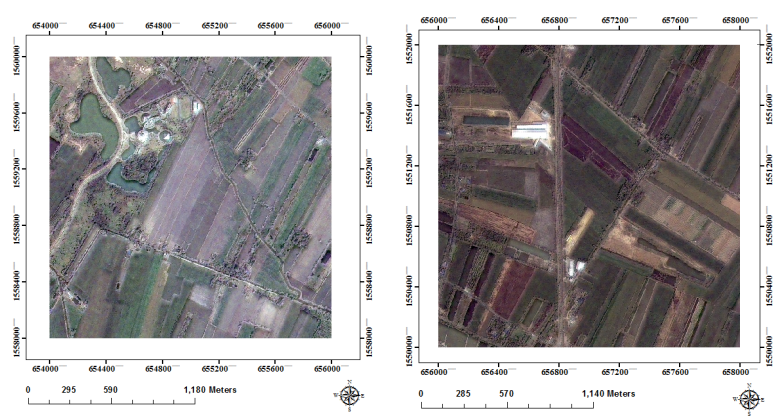

Figure 2: THEOS images of two sampled regions 
Table 1: Acquisition details of THEOS images

\begin{tabular}{ll}
\hline Image Type & Multispectral \\
\hline Resolution & 2 Meter \\
& Band 1 Blue $(0.452-0.512)$ \\
THEOS $(\mu \mathrm{m})$ & Band 2 Green $(0.533-0.590)$ \\
& Band 3 Red $(0.636-0.679)$ \\
Acquisition Date & Band 4 NIR $(0.851-0.879)$ \\
Source & February 1, 2012 \\
& Geo-Informatics and Space Technology \\
\hline
\end{tabular}
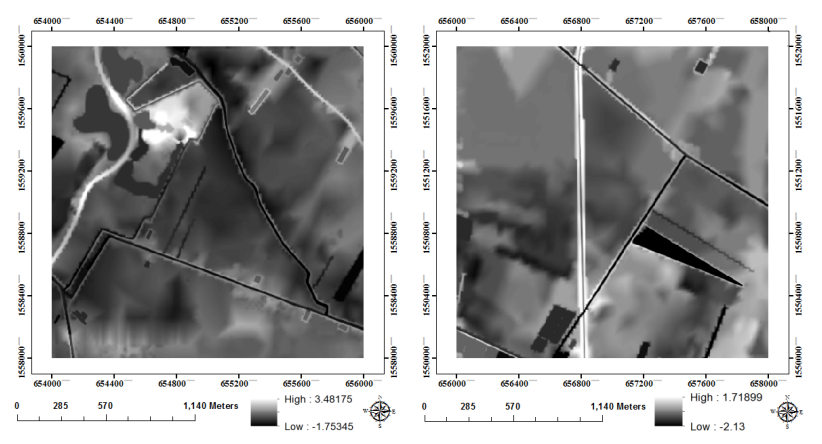

Figure 3: DSM data of two sample regions, in respect of Figure 2

\subsection{Spectral Indices Classification}

It has been concurred by existing studies that relying on any single index could not extract roads with sufficient accuracy. Unless some high-level vision algorithms were involved, there would remain some other misclassified structures in extractions. Maintaining that the proposed scheme could be readily incorporated into most RS software packages without having been dependent on extra programming intervention, this study thus selected three well accepted THEOS spectral indices, i.e., NDVI, SAVI and NDWI, whose expressions were provided in Table 2. These pixel-wise attributes would then be fed into SVM supervised learning to avoid having to manually nor programmatically configuring a threshold value. Otherwise, it would inevitably introduce inter- and intra-observers variabilities.

\subsection{Object Based Classification}

In the experiments, the proposed fusion method would be benchmarked against THEOS image analysis based on object based classification. With OBC, an image is segmented into regions or objects based on their homogeneity, instead of single pixel reflectance. Each thematic layer would then be reconstructed from a class described by similar congregated parameters. This study was compared with an OBC variant. Specifically, the scale, shape, and compactness, characterizing the size, weighting factor, and congregation were prescribed with 50, 0.2 and 0.8 , respectively. The resultant ensembles would subsequently be classified with SVM.

\subsection{Local Standard Deviation of DSM}

Following above literature review, we were able to conclude that road extraction based on individual or collective pixel reflectance or topographic data alone did not suffice. THEOS image has high spatial but limited spectral resolution. Derived spectral indices is thus not so much discernable as those from other multi-spectral modalities. DSM, on the other hand, does not contain any reflective attribute but is able to differentiate, at comparably high precision, various objects based on their elevations. It is worth pointing out that, unlike Digital Terrain Model (DTM), DSM data encode the height of objects covering the underlying terrain. Canopy, building roof top, river as well as road present on the same region could be effectively resolved. Thus far, there is no standard elevation value designated to any of these objects, depending of course on the terrain, upon which it is found. There exists, nonetheless, other topographic attributes typically adopted for terrain analyses, i.e., slope aspect, curvature, hillshade and viewshade. Based on a transportation criterion, stating that for engineering purposes, geometric variations along an automobile passage must not exceed some safety limit. Slope aspect and curvature had especially been preferred in road analyses. They were able to extract however only road boundaries but not its interior. Moreover, they may fail when the road height is of approximately same level as surrounding ground. This study thus exploited boundaryregion duality and expressed the relationship by using lo- 
Table 2: Spectral indices considered in this study

\begin{tabular}{lll}
\hline Indices & Expressions & References \\
\hline NDVI & $($ NIR- RED $) /(N I R+R E D)$ & {$[13]$} \\
SAVI & $($ NIR-RED $(1+L)) /(N I R+R E D+L)$ & {$[19]$} \\
NDWI & $($ Green-NIR) $/($ Green+NIR) & {$[21]$} \\
\hline
\end{tabular}

cal standard deviation (LSD) of DSM. It was hereby assumed as LSD approaches zero, roads may be differentiable from other land covers. In this study, LSD was calculated for any given location within a 10 meter radius $(5 \times 5$ pixel window, each pixel is of 2 meter resolution). Likewise, to avoid having to manually predefine a threshold (i.e., of being flat), LSDs were discriminated by means of a generic unsupervised ML, i.e., K-means algorithm.

\subsection{Machine Learning and Data Fusion}

It was previously anticipated that combining multispectral indices with DSM would help improving extraction accuracy, while ML played an important part in process automation. In this study, two ML methods were performed in parallel. Firstly, a supervised 2-class SVM was employed to classify road and non-road pixels based on NDVI, SAVI and NDWI, which were derived from THEOS data. The training set consisted of 1000 randomly sampled points, whose classes were known. Our preliminary experiments indicated that RBF kernel provided most accurate discrimination and hence was chosen. Secondly, an unsupervised 2-class K-means was similarly employed to discriminate roads and non-roads but based on LSD, derived from DSM based on the Euclidean metric. A total of 8 images were considered in the experiments. The extracted roads from both MLs were then fused in the subsequent step.

As anticipated, there remaining some other land covers such as buildings, with similar reflective indices, apart from roads in the SVM extracted results. These erroneous structures were however rid of in the K-means extraction, but other relatively flat objects were instead wrongly included. Generally, actual roads would exhibit in both classifications, Majority Voting was therefore employed for fusing these results. Specifically, a given pixel would be assigned to a road object class, only if it appeared in both SVM and K-means extractions.

\section{Results and Discussion}

Although spectral index based ML such as that by using SVM was extensively adopted in the literature, in the following experiment, we intended to show that when higher spatial resolution is desired, especially for road extraction, and thus if THEOS images were considered, discrimination capability of multi-spectral index alone did no longer suffice. This statement was elucidated by comparing extractions given by SVM classification (based on just spectral indices) and those given by the proposed data fusion method. These extraction results were both validated against the manually segmented ground truth, provided by the Department of Public Works and Town \& Country Planning), and the land use maps that was provided by the Land Development Department of Thailand. Figure 4-7(a) and $4-7$ (b) are the roads (shown as black pixels) extracted from images 1 to 4 , based on spectral indices and those by using the proposed method, respectively. Due to limited space, Figure $8(a-d)$ showed only those extracted from images 5-8, by using the proposed method. It is apparent that due to compromised spectral resolution, the former index based extraction had left some land covers other than road, e.g., buildings and water bodies. These false positives were mostly eliminated by the proposed fusion. However, leveled paved ground surrounded some buildings or local passage ways remained noticeable in Figure 6(b), 8(b) and 8(d). This was due to that those structures well conformed to both our spectral (THEOS) and geometrical (DSM) assumptions. Should they strictly were to be removed, intermediate post processing algorithm (e.g., polygons versus line segments, etc.) could be implemented, but they detailed treatment fell out of scope of this study.

In addition, road extraction accuracy was also evaluated and compared objectively by using six measures, i.e., precision (producer accuracy) and recall (user accuracy) of both road and non-road classes, overall accuracy and Kappa coefficient. To this end, the road pixels were validated against manually digitized references. For 8 images considered, averaged precisions (of road and non-road), recalls (of road and non-road), overall accuracy and Kappa 


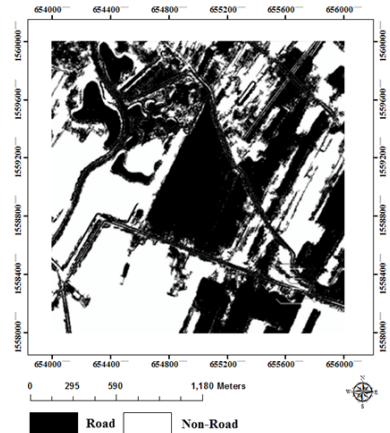

(a)

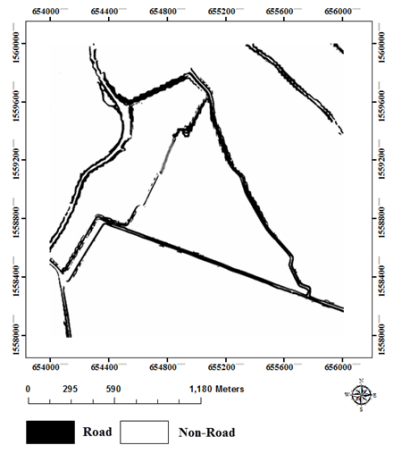

(b)
Figure 4: Road extractions from image No.1 by using multi-spectral indices (a) and the proposed method (b)

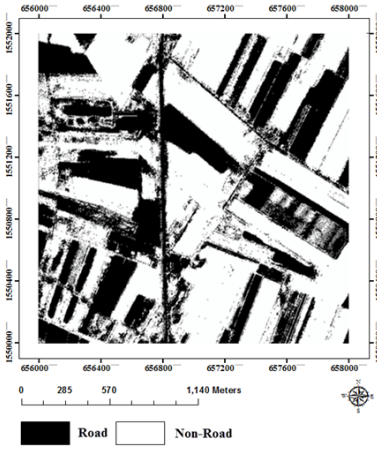

(a)

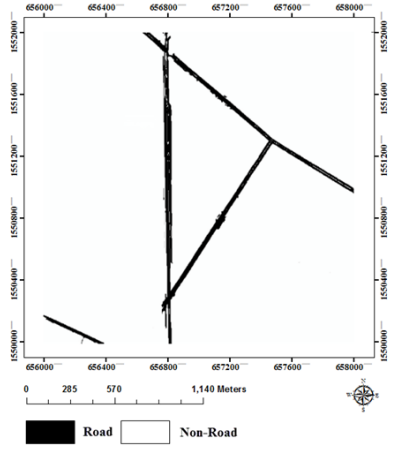

(b)
Figure 5: Road extractions from image No.2 by using multi-spectral Indices (a) and the proposed method (b)

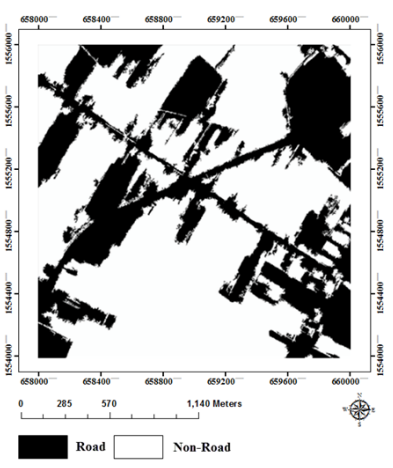

(a)

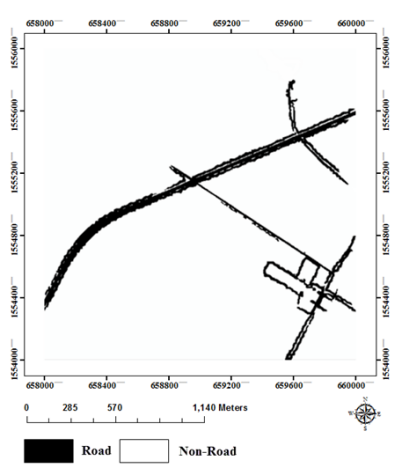

(b)
Figure 6: Road Extractions from image No.3 by using multi-spectral Indices (a) and the proposed method (b)

of spectral based SVM extraction were 11.13, 94.85, 80.65, $36.45,40.81$ percent and 0.05 , respectively, while those by means of the proposed method were 97.40, 97.66, 84.64, $99.72,97.78$ percent and 0.89 , respectively. The corresponding measures for each image are listed in Table 3 and Figure 10, for the comparison between the spectral based and the proposed methods.

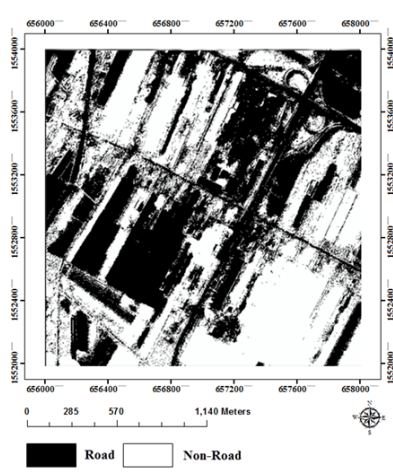

(a)

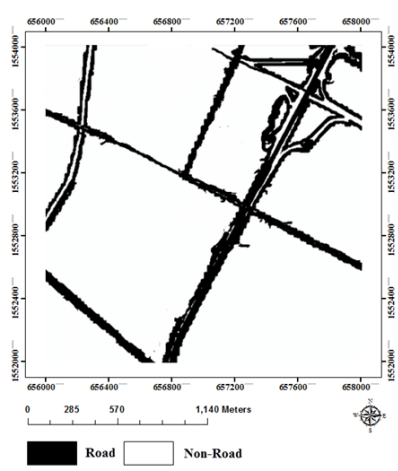

Figure 7: Road Extractions from image No.4 by using multi-spectral Indices (a) and the proposed method (b)

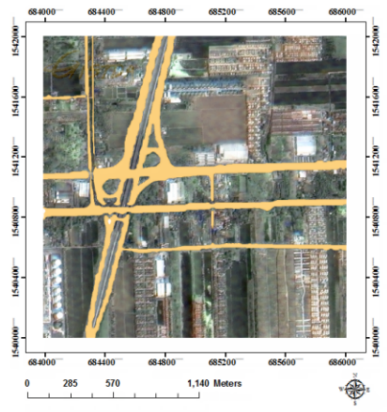

(a)

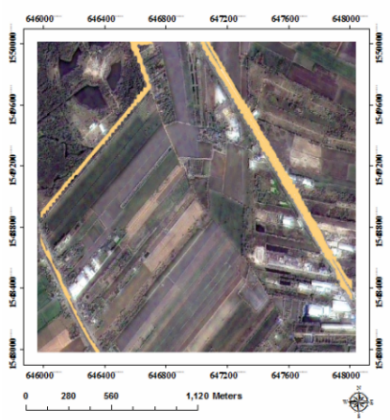

(c)

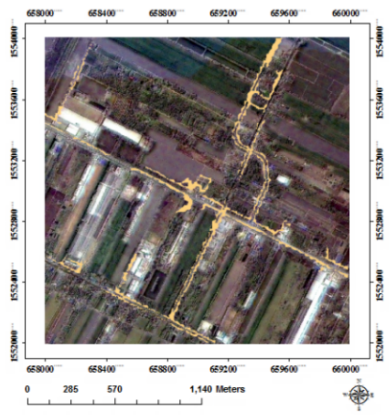

(b)

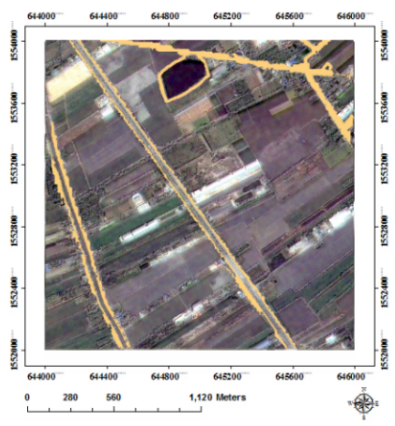

(d)
Figure 8: Additional examples of road extractions from Image No.5 -8 by using the proposed method. The results are overlaid on the corresponding THEOS image

Comparing the proposed method with a more recent $\mathrm{OBC}$ approach also highlights its merits. Although OBC could perform better than spectral based method, Figure 9 and Figure 10, indicate that it was inferior to the proposed method with the overall accuracy of only $57.86 \pm 14.28$. Visually, due to the absence of topographic inference, OBC had left some non-road as well as missed some road strictures, similar to that provided by spectral index based method. 
Table 3: Comparison of Overall Accuracy and Kappa coefficients and their arbitrate

\begin{tabular}{|c|c|c|c|c|}
\hline Img & Accuracy Spectral (mean \pm sd) & Proposed (mean \pm sd) & Kappa Spectral (mean \pm sd) & Proposed $(m e a n \pm s d)$ \\
\hline 1 & 37.44 & 96.65 & 0.07 & 0.82 \\
\hline 2 & 44.10 & 98.92 & 0.05 & 0.90 \\
\hline 3 & 59.04 & 98.65 & 0.11 & 0.91 \\
\hline 4 & 44.67 & 93.02 & 0.11 & 0.78 \\
\hline 5 & 28.95 & 99.83 & 0.01 & 0.97 \\
\hline 6 & 38.61 & 97.84 & 0.02 & 0.86 \\
\hline 7 & 47.81 & 97.81 & 0.01 & 0.89 \\
\hline 8 & 25.85 & 99.51 & 0.01 & 0.98 \\
\hline avg & $40.81 \pm 10.60$ & $97.78 \pm 2.18$ & $0.05 \pm 0.04$ & $0.89 \pm 0.07$ \\
\hline
\end{tabular}

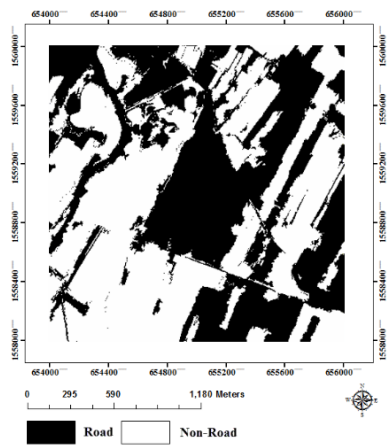

(a)

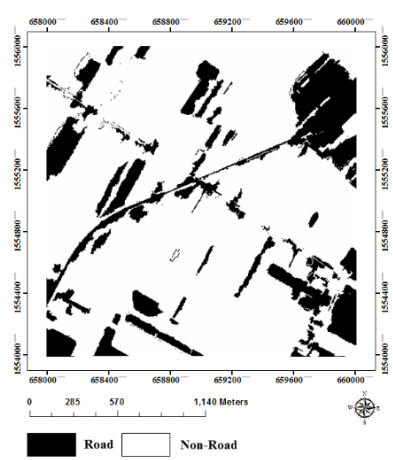

(c)

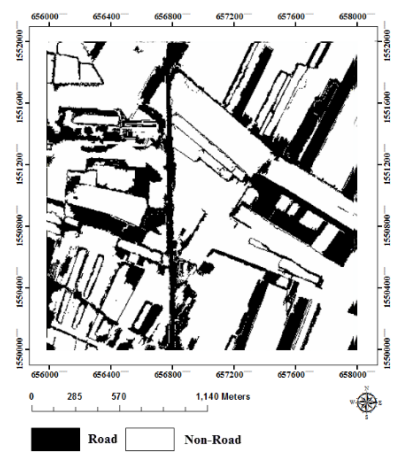

(b)

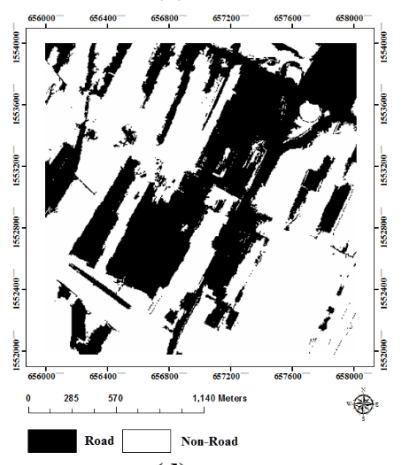

(d)
Figure 9: Road extractions from image No.1-4 by using object based classification

It is thus safe to say that $\mathrm{OBC}$ is unsuitable for such task, based on THEOS images.

Unlike other multi-spectral modalities with lower resolutions, THEOS has much poorer band separation, and hence without any other contextual/ topographic information, it was outperformed by the proposed methods in terms of accuracies by $50-80 \%$ (except for small discrepancies in precision and recall of the non-road class). In general, the overall accuracy of the proposed method was much higher at $97.78 \pm 2.18$ percent. Moreover, its Kappa of 0.89 indicated that the extracted roads corresponded well to those in the ground truth references. To assess the sensitivity and specificity of both methods, Figure 12 compared their TP, TN, FP and FN rates, respectively, per images 1-8 (see graph labels for detailed annotations). More specifically, TP and FP mean the pixels were assigned to the road class, while they were in fact road and non-road pixels, respectively. Likewise, TN and FN mean the non-road pixels were assigned correctly and incorrectly as such. The TP and FP rates and TN and FN rates were calculated by the ratio between the values and their relevant class sums, i.e., $(\mathrm{TP}+\mathrm{FP})$ and $(\mathrm{TN}+\mathrm{FN})$, respectively. It was evident from these plots that the specificities (TP rates) and sensitivities (TN rates) given by the proposed method for all images were consistently higher than its counterparts. To elevate limited discrimination capability of reflective spectra in high spatial resolution images, geometrical derivative may be incorporated and have ambiguous structures removed by trivial fusion. This was clearly manifested in both visual inspection and low FN and FP rates of the results obtained from the proposed method.

To better demonstrate qualitatively the performance of the proposed method, it was also compared with roads of topographic maps. Due to its reflective properties, only paved road was able to be extracted. Other unhandled roads were dirt road, gravel road and laterite or murram road. Two types of roads considered in the subsequent illustrations are highway/ main-street (Type 1) and avenue (Type 2), whose actual widths were not less than 4 meters. The resultant extractions were compared with both respective satellite images (Figures 13 and 14) and the topographic maps (Figures 15 and 16) provided by Google Earth. Figure 13 shows both roads of Type 1 (orange) and 2 (blue), whose names are Bangkok Western Outer Ring Highway and Wat Chan Tharam Street, respectively. The insets display the corresponding perspective view of the actual roads. Figure 14 shows similar extractions but of only Type 2 (white) roads. The road labeled with number 

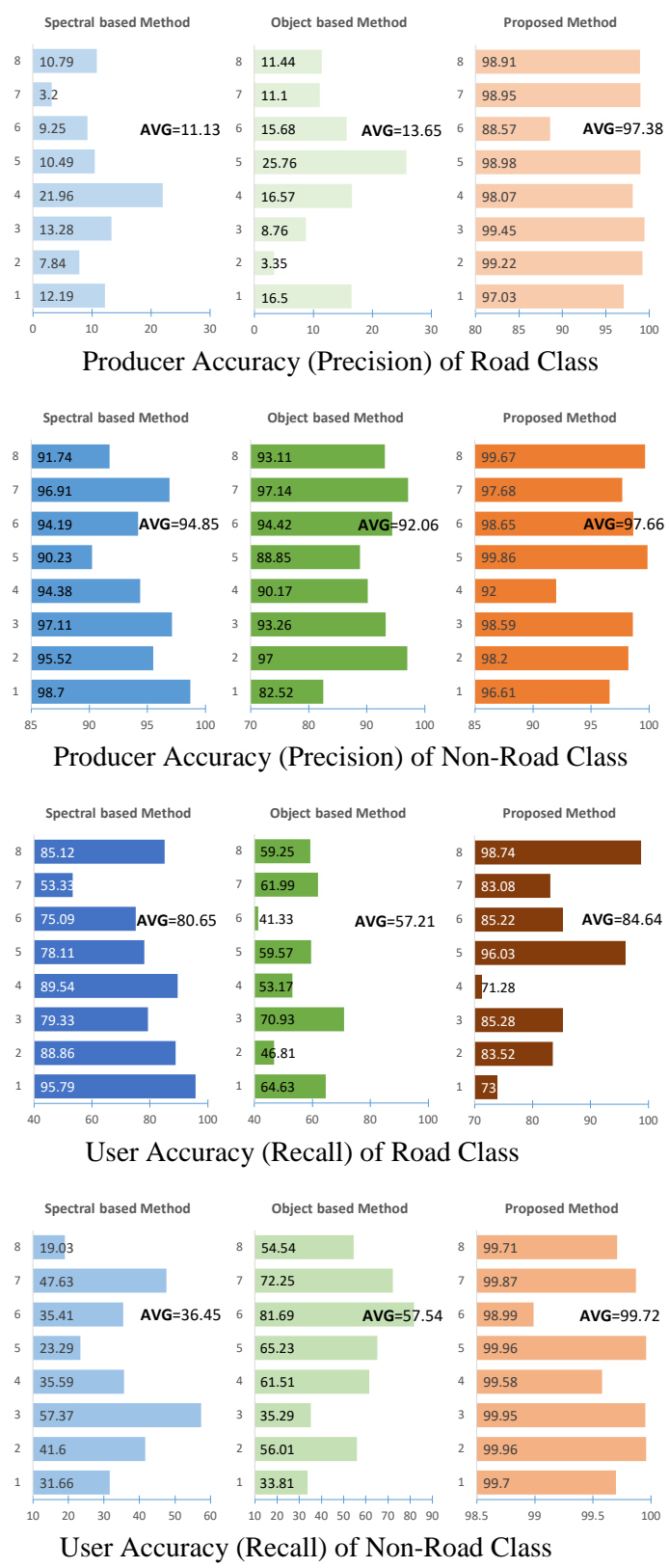

Figure 10: Comparison of Precisions and Recalls of roads and nonroads in each image $(1-8)(y$-axis). The values are expressed in percent ( $\mathrm{x}$-axis). For clarity, the $\mathrm{x}$-axis range was adjusted per each category

1 is an unnamed road, located in Bang Toei sub-district, Pathumthani, while that labelled with number 2 is Khu Khwang 10 alley. It is clear from both figures that the extracted roads well corresponded to their actual Google satellite maps. Figure 15(a) illustrates the extractions overlaid on the Google topographic map, while Figure 15(b) and (c) zoom into the Bangkok Western Outer Ring Highway and Wat Chan Tharam Street, respectively. Figure 16
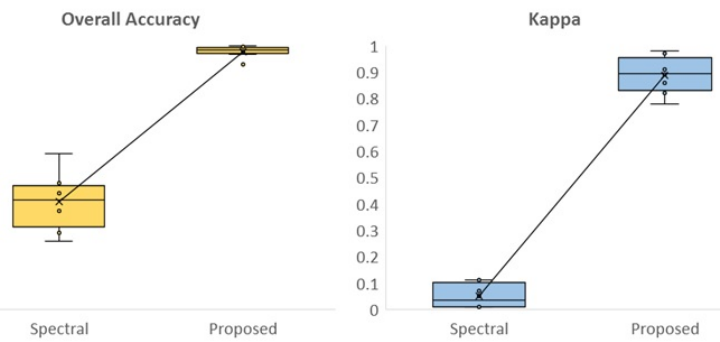

Figure 11: Comparison of Overall Accuracy and Kappa coefficients and their arbitrate
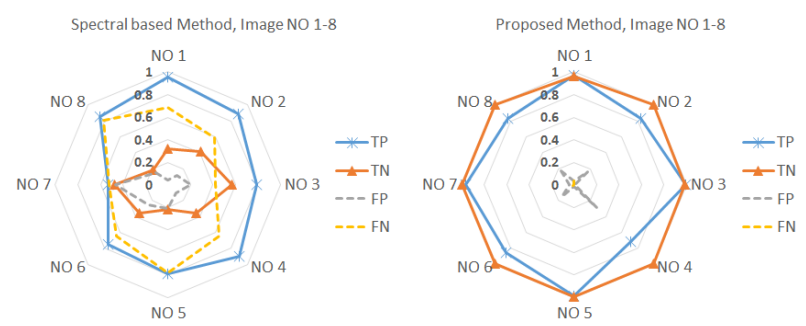

Figure 12: Specificity and sensitivity of road extractions from image No.1-8 (in each angle) by using multi-spectral indices based (left) and the proposed method (right)

shows similar topographic overlays of smaller road extractions, corresponding to Figure 14.

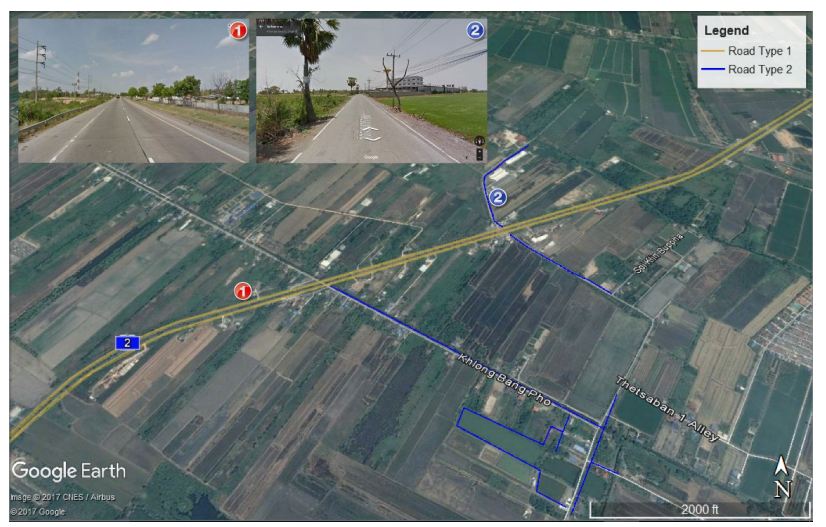

Figure 13: Two types of extraction results overlaid on the Google topographic map

In comparison with other existing works on road extractions, the proposed method has overcome several previous and limitations. They include but not limited to having to rely on user to initialize the estimation [4], depending on threshold that varies from one study area to other [22, 23], being unable to removed other urban structures $[22,23,34,35]$ and being suffered from relatively low accuracy [22, 23, 34, 35]. More detailed comparison with the baseline techniques (e.g., those based on single 


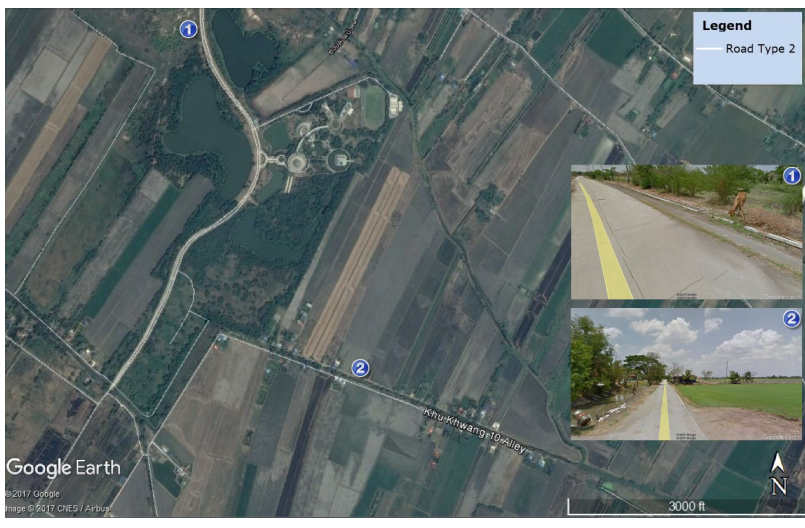

Figure 14: Another example of Type 2 roads overlaid on the Google topographic map

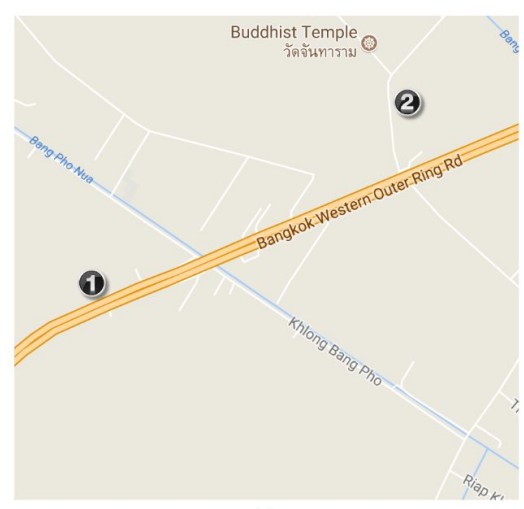

(a)

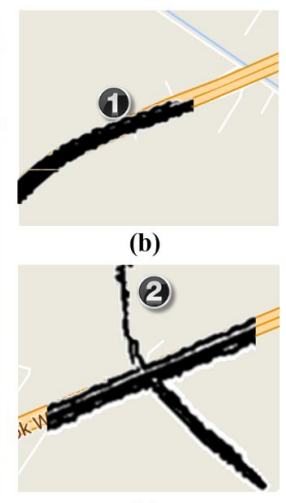

(c)
Figure 15: Google topographic map (a) and corresponding road extractions from the areas number 1 (b) and 2 (c), respectively

aerial and DSM image), the proposed method has 10\% higher accuracy with 0.25 higher Kappa. The proposed method was also able to more satisfactorily remove nonroad pixels than previously possible [16]. More recent OBC method [34, 35], with which the proposed method was also benchmarked, yielded less accuracy than the proposed method by approximately 4-5\%. It also missed some road pixels while leaving some urban objects misclassified. Moreover, in terms of ML strategy, a conventional Fuzzy-C-means classification on THEOS images [24] was unable to reach a comparable results, i.e., with by $25 \%$ lower accuracy. It is therefore safe to conclude that fusion of multi-spectral indices and DSM local statistics, with SVM and K-means classification, could overcome impeding factors, previously admitted.

The proposed method is suitable for road extraction based on optical satellite images that consist of NIR2 bands. It also at least requires DSM information of the corresponding area for LSD calculation to further enhance extraction performance. Without NIR band, it is reasonable to believe that, fusing $\mathrm{OBC}$ result with topographic data in the similar manner might as well increase the extraction accuracy, but its elucidation fell out of scope of this paper and opened for prospective investigations.

\section{Conclusion}

Spectral indices based geographical object classification has been one of active research areas in remote sensing. Thus far, cautions should be observed when, in some modalities, their spectral resolution is not comparable to their spatial one. This maybe usually the case when detailed geometrical analysis (such as of river branches and roads, etc.) is required. Increasing spatial resolution by up-sampling or opting for alternative acquisitions does not necessarily guarantee the success, using for example, the same indices. We have so far reported such case when THEOS is considered for detailed road extraction. It was evident from the experiments that based on NDVI, SAVI and MNDWI alone, even when used with the state-of-the art ML algorithm, remained unable to correctly extract road structures.

To address this issue, we presented a data fusion based method for accurate and robust road extraction in a high spatial resolution multi-spectral THEOS satellite images. To this end, assumptions on particular topographic properties, i.e., local standard deviation, derived from DSM data were imposed on the extraction result by fusion. Unlike the other approaches in the literature, where higher level computer vision algorithms (e.g., morphology, Hough transform, shape analysis, etc.) were typically called for, the proposed scheme made used of only off-the-shelf elements, already available in most open source/ commercial software packages. This has enabled it to be easily deployable in a typical RS laboratory setting, with less programmatically involved. Both visual and numerical assessments reported herein exhibited that the resultant road extractions was satisfactory, conforming to the ground truth references. Depending on analytical purposes, however, advanced computer vision techniques may be concatenated to determine, for instance, topologically valid road networks. Figure 17 illustrates one such example, where trivial thinning was applied to extracted road pixels to assist their perceptual grouping. Detailed treatment of those post-processing techniques fell out of scope of the current presentation and readers are referred to studies already mentioned in the literature.

Another aspect worth investigated is an optimal choice of imaging modalities for this application. For 


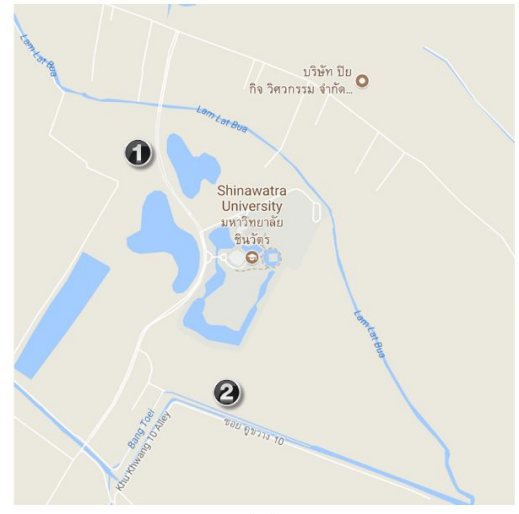

(a)

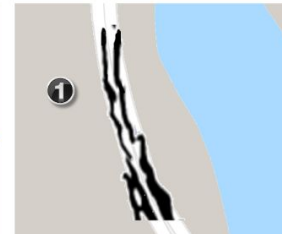

(b)

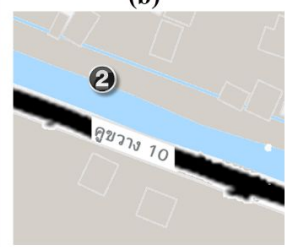

(c)
Figure 16: Google topographic map (a) and corresponding road extractions from the areas number 1 (b) and 2 (c), respectively

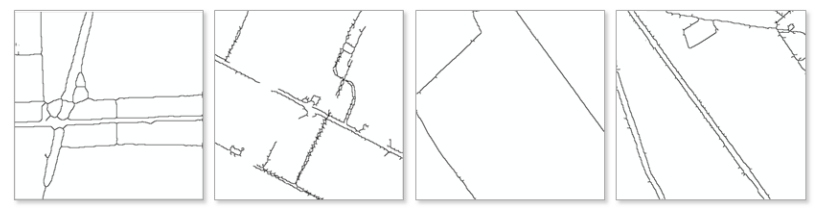

Figure 17: Examples of possible extended road extraction, where trivial thinning was applied to extracted road pixels to assist their perceptual grouping

examples, WorldView, IKONOS and QuickBird all are of higher spatial and spectral resolution than THEOS and thus could offer better road extraction. In addition, more advanced Artificial Intelligent (AI) schemes may be explored so as to better recognize other geographical structures with similar reflective properties, such as building, river and road.

Acknowledgement: The authors would like to thank the Department of Public Works and Town and Country Planning, USGS website, Geo-Informatics, Google Inc, and Space Technology Development Agency, Public Organization and the Land Development Department of Thailand for providing the remotely sensed data (THEOS) used in preparation of the paper.

\section{References}

[1] Asmus V.V., Buchnev A. A., Pyatkin V.P., Controlled classification of Earth remote sensing data. Optoelectronics, Instrumentation and Data Processing, 2008, 44(4), 331-336

[2] Blaschke T., Object based image analysis for remote sensing. ISPRS journal of photogrammetry and remote sensing, 2010, 65(1), 2-16
[3] Lv Q., Dou Y., Niu X., Xu J., Xu J., Xia F., Urban land use and land cover classification using remotely sensed SAR data through deep belief networks. Journal of Sensors, 2015, 2015(1), 1-10, DOI: $10.1155 / 2015 / 538063$

[4] Desai V.P., Vala H., Survey on methods of road extraction using satellite image. International Journal of Engineering Research and Technology, 2014, 3(11), 1422-1424

[5] Furuto T., Yamada H., Kakumoto S., Development of evacuation support system in the areas affected by multi-hazards. In Proceedings of the 14 th World Conference on Earthquake Engineering, Beijing, China, 2008, 1-6

[6] Shimura Y., Yamamoto K., Method of searching for earthquake disaster evacuation routes using multi-objective GA and GIS. Journal of Geographic Information System, 2014, 6(5), 492-525, DOI: $10.4236 /$ jgis.2014.65042

[7] Bosona T., Nordmark I., Gebresenbet G., and Ljungberg D., GISbased analysis of integrated food distribution network in local food supply chain. International Journal of Business and Management, 2013, 8(17), 13-34, DOI: 10.5539/ijbm.v8n17p13

[8] Kim J.G., Han D.Y., Yu K.Y., Kim Y.I., Rhee S.M., Efficient extraction of road information for car navigation applications using road pavement markings obtained from aerial images, Canadian Journal of Civil Engineering, 2006, 33(10), 1320-1331, DOI: 10.1139/106-069

[9] Jin X., Davis C.H., Automated building extraction from highresolution satellite imagery in urban areas using structural, contextual, and spectral information. EURASIP Journal on Advances in Signal Processing, 2005, 2005(14), 1-11, DOI: 10.1155/asp.2005.2196

[10] Liu Z., Cui S., Yan Q., Building extraction from high resolution satellite imagery based on multi-scale image segmentation and model matching. In Earth Observation and Remote Sensing Applications, EORSA 2008. International Workshop, IEEE, 2008, 17 DOI: https://doi.org/10.1109/eorsa.2008.4620321

[11] Cui W.H., Feng X., Qin K., The pixel rectangle index used in object based building extraction from high resolution images, in 35th International Symposium on Remote Sensing of Environment (ISRSE35), 2014, 1-7, DOI: 10.1088/1755-1315/17/1/012233

[12] Zha Y., Ni S.X., Yang S., An effective approach to automatically extract urban land-use from TM imagery. Journal of Remote Sensing-BEIJING, 2003, 7(1), 37-40

[13] Rouse Jr J., Haas R.H., Schell J.A., Deering D.W., Monitoring vegetation systems in the Great Plains with ERTS, in Third ERTS Symposium, 1973, 309-317

[14] Shackelford A.K., Davis C.H., Fully automated road network extraction from high-resolution satellite multispectral imagery. In Geoscience and Remote Sensing Symposium, 2003. IGARSS'03. Proceedings. 2003 IEEE International, 2003, 461463, DOI: 10.1109 /igarss.2003.1293809

[15] Mhangara P., Odindi J., Kleyn L., Remas H., Road extraction using object oriented classification. http://africageodownloads. info/058_mhangara_odindi_kleyn_remas.pdf (Last accessed 01/04/2016).

[16] Rodríguez-Cuenca B., de Agirre Á.M., Alonso M.C., del Val A., Road extraction method at the pixel and object level using high resolution images and LIDAR data with evidence theory. In ESA Special Publication, 2013, 722(1), 269-274

[17] Prakash T., Comandur B., Chang T., Elfiky N., Kak A.A., Generic road-following framework for detecting markings and objects in satellite imagery. IEEE Journal of Selected Topics in Applied 
Earth Observations and Remote Sensing, 2015, 8(10), 47294741, DOI: $10.1109 /$ jstars.2015.2495142

[18] Lak A.M., Zoej M.J.V., Mokhtarzade M., A new method for road detection in urban areas using high-resolution satellite images and Lidar data based on fuzzy nearest-neighbor classification and optimal features. Arabian Journal of Geosciences, 2016, 9(5), 1-11. DOI: 10.1007/s12517-016-2374-1

[19] Huete A.R., A soil-adjusted vegetation index (SAVI). Remote sensing of environment, 1988, 25, 295-306, DOI: 10.1016/00344257(88)90106-x

[20] Karaman E., Cinar U., Gedik E., Yardemci Y., Halici U., A new algorithm for automatic road network extraction in multispectral satellite images. Proceedings of the 4th GEOBIA, 2012, 455-459

[21] Mcfeeters S.K., The use of normalized difference water in$\operatorname{dex}(\mathrm{NDWI})$ in the delineation of open water features, International Journal of Remote Sensing, 1996, 17(7), 1425-1432, DOI: $10.1080 / 01431169608948714$

[22] Shahi K., Shafri H.Z., and Taherzadeh E., A novel spectral index for automatic shadow detection in urban mapping based on WorldView-2 satellite imagery. International Journal of Computer, Control, Quantum and Information Engineering, 2014, 8(10), 27-33

[23] Shahi K., Shafri H.Z., Taherzadeh E., Mansor S., Muniandy R., A novel spectral index to automatically extract road networks from WorldView-2 satellite imagery. The Egyptian Journal of Remote Sensing and Space Science, 2015, 18(1), 27-33, DOI: 10.1016/j.ejrs.2014.12.003

[24] Sangpradid S., Sarapirome S., Road extraction from pansharppened THEOS images. Journal of Remote Sensing and GIS Association of Thailand, 2014, 15(2), 12-17

[25] Herold M., Gardner M.E., and Roberts D.A., Spectral resolution requirements for mapping urban areas. Geoscience and Remote Sensing, IEEE Transactions on, 2003, 41(9), 1907-1919, DOI: $10.1109 /$ tgrs.2003.815238

[26] Carleer A., Wolff E., VHR data region-based classification possibilities in the framework of Control with Remote Sensing of European CAP. In 31st International Symposium of Remote Sensing on Environment, 2005, 1-4, DOI: 10.1117/12.627641

[27] Lu Y.H., Trinder J.C., Kubik K., Automatic building detection using the Dempster-Shafer algorithm. Photogrammetric Engineering \& Remote Sensing, 2006, 72(4), 395-403, DOI: 10.14358/pers.72.4.395

[28] Puttinaovarat S., Horkaew P., Khaimook K., Polnigongit W., Adaptive hydrological flow field modeling based on water body extraction and surface information, Journal of Applied Remote Sensing, 2015, 9(1), 095041-1-095041-22, DOI: 10.1117/1.jrs.9.095041

[29] Lam L. Suen C.Y., Application of majority voting to pattern recognition: an analysis of its behavior and performance, Systems, Man and Cybernetics, Part A: Systems and Humans, IEEE Transactions, 1997, 27(5), 553-568, DOI: 10.1109/3468.618255

[30] Hégarat-Mascle L., Bloch I., Vidal-Madjar D., Application of Dempster-Shafer evidence theory to unsupervised classification in multisource remote sensing. Geoscience and Remote Sensing, IEEE Transactions, 1997, 35(4), 1018-1031, DOI: $10.1109 / 36.602544$

[31] Samadzadegan F., Hahn M., Bigdeli B., Automatic road extraction from LIDAR data based on classifier fusion. In Urban Remote Sensing Event 2009 Joint, IEEE, 2009, 1-6, DOI: $10.1109 /$ urs.2009.5137739
[32] He C., Shi P., Xie D., Zhao Y., Improving the normalized difference built-up index to map urban built-up areas using a semiautomatic segmentation approach. Remote Sensing Letters, 2010, 1(4), 213-221, DOI: 10.1080/01431161.2010.481681

[33] Patel N., Mukherjee R., Extraction of impervious features from spectral indices using artificial neural network. Arabian Journal of Geosciences, 2015, 8(6), 3729-3741, DOI: $10.1007 /$ s12517014-1492-x

[34] Wang P., Zhai Z., Fu H., Zhao Z., Automatic Extraction of Road Information Using the Object-Oriented Technology. The Open Automation and Control Systems Journal, 2015, 7, 1842-1849, DOI: $10.2174 / 1874444301507011842$

[35] Rizvi I.A., Mohan B.K., Object-oriented method for automatic extraction of road from High Resolution Satellite Images. Iranian Journal of Earth Sciences, 2010, 2(1), 55-62.

[36] Maboudi M., Amini J., Object based segmentation effect on road network extraction from satellite images. In Proceedings of the 36th Asian Conference on Remote Sensing, Manila, Philippines, 2015. 19-23

[37] Kumar M., Singh R.K., Raju P.L.N., Krishnamurthy Y.V.N., Road network extraction from high resolution multispectral satellite imagery based on object oriented techniques. ISPRS Annals of the Photogrammetry, Remote Sensing and Spatial Information Sciences, 2014, 2(8), 107, DOI: 10.5194/isprsannals-ii-8107-2014

[38] Miao Z., Shi W., Gamba P., Li Z., An object-based method for road network extraction in VHR satellite images. IEEE Journal of Selected Topics in Applied Earth Observations and Remote Sensing, 2015, 8(10), 4853-4862, DOI: 10.1109/jstars.2015.2443552

[39] Li Y., Lin Z.L., Shimamura H., Integrated method of building extraction from Digital Surface Model and imagery. The International Archives of the Photogrammetry, Remote Sensing and Spatial Information Sciences, 2008, 37, 81-86

[40] Brédif M., Tournaire O., Vallet B., Champion N., Extracting polygonal building footprints from digital surface models: a fully-automatic global optimization framework. ISPRS Journal of Photogrammetry and Remote Sensing, 2013, 77, 57-65, DOI: 10.1016/j.isprsjprs.2012.11.007

[41] Song X., Tang G., Li F., Jiang L., Zhou Y., Qian K., Extraction of loess shoulder-line based on the parallel GVF snake model in the loess hilly area of China. Computers \& Geosciences, 2013, 52, 11-20, DOI: 10.1016/j.cageo.2012.08.014

[42] Herumurti D., Uchimura K., Koutaki G., Uemura T., Urban road network extraction from very high resolution RGB aerial images and DSM data. In 34th Asian Conference on Remote Sensing, 2013, 725-73, DOI: 10.1109/sitis.2013.24

[43] Herumurti D., Uchimura K., Koutaki G., Uemura T., Urban road extraction based-on morphological operations and radon transform on DSM data. ITE Transactions on Media Technology and Applications, 2014, 2(3), 277-286, DOI: 10.3169/mta.2.277

[44] Matkan A.A., Hajeb M., Sadeghian S., Road extraction from LIDAR data using support vector machine classification. Photogrammetric Engineering \& Remote Sensing, 2014, 80(5), 409422, DOI: $10.14358 /$ pers.80.5.409

[45] Turker M., Koc-San D., Building extraction from high-resolution optical spaceborne images using the integration of support vector machine (SVM) classification, Hough transformation and perceptual grouping.International Journal of Applied Earth Observation and Geoinformation, 2015, 34, 58-69, DOI: 10.1016/j.jag.2014.06.016 
[46] Palamuleni L.G., Ndou N.N., Developing remote sensing methodology to distinguish urban built-up areas and bare land in Mafikeng town, South Africa. In Geoscience and Remote Sensing Symposium (IGARSS), 2014 IEEE International, 2014, 2205-2208, DOI: 10.1109/igarss.2014.6946906

[47] Pisani R., Riedel P., Ferreira M., Marques M., Mizobe R., Papa J., Land use image classification through optimum-path forest clustering. In Geoscience and Remote Sensing Symposium (IGARSS) 2011 IEEE International, 2011, 826-829, DOI: 10.1109/igarss.2011.6049258

[48] Sowmya B., Sheelarani B., Land cover classification using reformed fuzzy C-means. Sadhana, 2011, 36(2), 153-165, DOI: 10.1007/s12046-011-0018-4
[49] Wang Y., Li X., Zhang L., Zhang W., Automatic road extraction of urban area from high spatial resolution remotely sensed imagery. Int. Arch. Photogrammetry, Remote Sen. Spatial Inform. Sci, 2008, 86

[50] Maurya R., Gupta P.R., Shukla A.S., Road extraction using kmeans clustering and morphological operations. In Image Information Processing (ICIIP), 2011 International Conference, 2011, 1-6, DOI: 10.1109/iciip.2011.6108839 